\title{
$\mathrm{M}|\mathrm{R}| \mathrm{S}$ Internet Journal Nitride Semiconductor Research
}

\section{Current limitation after pinch-off in AIGaN/GaN FETs}

\author{
R. Dietrich ${ }^{1}$, A. Wieszt ${ }^{1}$, A. Vescan ${ }^{1}$, H. Leier ${ }^{1}$, Joan M. Redwing ${ }^{2}$, Karim S. Boutros ${ }^{2}$, K.

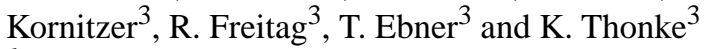 \\ ${ }^{1}$ DaimlerChrylser AG, Ulm, Germany, \\ ${ }^{2}$ Epitronics/ATMI, \\ ${ }^{3}$ Universität Ulm, Abteilung Halbleiterphysik, Germany,
}

(Received Thursday, January 27, 2000; accepted Wednesday, March 22, 2000)

Piezoelectric $\mathrm{AlGaN} / \mathrm{GaN}$ FETs on $\mathrm{SiC}$ with high carrier mobility have been fabricated yielding $\mathrm{I}_{\mathrm{DS}}=450 \mathrm{~mA} / \mathrm{mm}$ and $\mathrm{g}_{\mathrm{m}}=200 \mathrm{mS} / \mathrm{mm}$. In the on-state, under UV-illumination, the devices sustain a drain voltage of $\mathrm{V}_{\mathrm{DS}}=49 \mathrm{~V}$, corresponding to a power dissipation of $26.5 \mathrm{~W} / \mathrm{mm}$. On turn-on of the device from the pinch-off state, a significant delay in the drain current build-up is observed. This effect depends on the pinch-off time and the pinch-off voltage and can be removed by either a brief $\mathrm{UV}$-illumination or $\mathrm{a} \mathrm{V}_{\mathrm{DS}}>25 \mathrm{~V}$ applied in the on-state. The drain current transients are characterized by a relaxation time $\tau$, which is in the order of several hundred seconds. From the temperature dependence of $\tau$, an activation energy of about $280 \mathrm{meV}$ and a capture cross section of $4.4 \cdot 10^{-18} \mathrm{~cm}^{2}$ were determined. The devices show pronounced persistent photoconductivity (PPC) and the drain current $I_{D}$ is sensitive to illumination.

\section{Introduction}

Electronic devices based on $\mathrm{GaN}$ have attracted growing attention during the past years. This is mainly due to the promising material properties of $\mathrm{GaN}$ with respect to performance improvements for high-frequency power modules. To date, remarkable AlGaN/GaN FET-device results have been published with $\mathrm{f}_{\max } \approx 140 \mathrm{GHz}$ for small signals [1] and $6.9 \mathrm{~W} / \mathrm{mm}$ output power density at $10 \mathrm{GHz}$ [2]. To further increase the performance and provide reliable operation it is important to investigate possible limiting factors. In this report, we discuss a drain current delay effect and its removal by either a UV-illumination or by applying a high $\mathrm{V}_{\mathrm{DS}}$ to the device in the on-state .

\section{Device fabrication}

The epitaxial layer structure of the the FETs was grown in a vertical MOCVD reactor at $1100^{\circ} \mathrm{C}$. A $100 \mathrm{~nm}$ AlN nucleation layer, a $3 \mu \mathrm{m} \mathrm{GaN}$ buffer and a $23 \mathrm{~nm}$ $\mathrm{Al}_{0.3} \mathrm{GaN}$ top layer were deposited on a $(0001) \mathrm{n}^{+}-\mathrm{SiC}$ substrate. No dopants were used. The undoped $\mathrm{GaN}$ buffer was deposited at a growth rate of $2 \mu \mathrm{m} / \mathrm{h}$ and a V/ III ratio of about 4000 . These growth conditions resulted in a smooth surface morphology. Undoped layers grown under these conditions are typically highly resistive $\left(\mathrm{n}<5 \cdot 10^{15} / \mathrm{cm}^{3}\right)$ which is indispensable for achieving good pinch-off behaviour of electronic devices.

Mesa isolation was achieved by RIE dry etching with a photoresist mask. The ohmic metallisation consisted of a Ti/Al/Ni/Au layer structure. Pt/Au was evaporated for the gate contacts.

Hall measurements at RT yield mobilities of $\mu=1900$ $\mathrm{cm}^{2} / \mathrm{Vs}$ and sheet carrier concentrations of $\mathrm{n}_{\mathrm{s}}=3.5 \cdot 10^{12}$ / $\mathrm{cm}^{2}$. For a fully strained AlGaN layer with an $\mathrm{Al}$ mole fraction of $30 \%$, a piezoelectrically induced sheet charge of above $10^{13} / \mathrm{cm}^{2}$ is expected [3]. The discrepancy between the measured and the expected carrier concentration may arise from relaxation of the $\mathrm{Al}_{0.3} \mathrm{GaN}$ layer or from trapping effects. The ohmic contact resistance $\mathrm{R}_{\mathrm{c}}$ varies between 0.6 and $0.9 \Omega \mathrm{mm}$ after alloying at $900^{\circ} \mathrm{C}$ in $\mathrm{N}_{2}$ ambient.

\section{Results and discussion}

At a gate-length of $1 \mu \mathrm{m}$, the FETs yield a saturation current of $450 \mathrm{~mA} / \mathrm{mm}$ and a maximum transconductance of $200 \mathrm{mS} / \mathrm{mm}$ measured with a HP4145 parameter analyzer. Good ohmic contacts and the high electron mobility provide a low knee voltage of $3 \mathrm{~V}$. The ratio $I_{D}($ on $) / I_{D}$ (off) is larger than $10^{6}$ due to extremely low 
gate-leakage currents. In a two terminal setup, gatedrain breakdown occurs at $110 \mathrm{~V}$ and is independent of the gate-drain spacing from 1.4 to $6 \mu \mathrm{m}$. The mesa isolation is about $40 \mathrm{G} \Omega$ measured at $30 \mathrm{~V}$ between two different mesas. As the etch depth is only $100 \mathrm{~nm}$, this result indicates that the buffer is highly resistive.

Figure 1 shows the output characteristic of a $20 \mu \mathrm{m}$ device. The FET sustains its maximum drain current of $540 \mathrm{~mA} / \mathrm{mm}$ up to $\mathrm{V}_{\mathrm{DS}}=49 \mathrm{~V}$ (Figure 1). The corresponding power dissipation of $26.5 \mathrm{~W} / \mathrm{mm}$ did not destroy the device due to the excellent thermal conductivity of the $\mathrm{SiC}$ substrate. This measurement was carried out under UV-illumination with a Hg-lamp. In order to obtain a well defined state of the device these conditions were chosen since the device performance is dependent on the previous biasing conditions, as discussed below.

Measuring the output characteristic of the FETs under room light, a drastic current reduction is observed when the device is measured for a second time, $10 \mathrm{~s}$ after the first measurement. Figure 2 shows the topmost traces of the output characteristics measured for the first time (device was at equilibrium), 10 seconds later and a third time after a brief UV-illumination. Obviously, the current reduction can be removed by incident photons. This current reduction can equally be removed by applying a drain voltage $\mathrm{V}_{\mathrm{DS}}>25 \mathrm{~V}$. Before measuring this trace, the device was pinched off at $\mathrm{V}_{\mathrm{GS}}{ }^{0}=-10 \mathrm{~V}$ for 5 min to get a pronounced current reduction (dotted line). The sudden removal of the current reduction at $\mathrm{V}_{\mathrm{DS}}>25$ $\mathrm{V}$ can be explained by field enhanced carrier emission from traps. At $\mathrm{V}_{\mathrm{DS}}=25 \mathrm{~V}$ the electrical field between drain and gate is at least $20 \mathrm{MV} / \mathrm{m}$.

It is observed that the current reduction is influenced by the "history" of the gate voltage. Hence, the transients of the drain current $\mathrm{I}_{\mathrm{D}}$ at fixed drain and gate biases were studied for different gate pre-biasing conditions. This pre-biasing is denoted by the superscript ${ }^{0}$. Figure 3 shows the results of $I_{D}(t)$. Before $t=0$, the device was pre-biased for $5 \mathrm{~min}$ at the $\mathrm{V}_{\mathrm{GS}}{ }^{0}$ value indicated in the figure and at $\mathrm{V}_{\mathrm{DS}}{ }^{0}=100 \mathrm{mV}$. At $\mathrm{t}=0$, the device was turned on $\left(\mathrm{V}_{\mathrm{GS}}=0 \mathrm{~V}\right)$ and $\mathrm{I}_{\mathrm{D}}$ was recorded for $20000 \mathrm{~s}$. A low $V_{D S}$ value was applied in order not to influence the current recovery process by the applied drain voltage. As the drain current is very sensitive to incident light, the whole measurement was carried out in the dark and prior to each measurement the device was kept for 5 hours at zero bias in the dark to achieve approximately thermodynamic equilibrium. The current reduction effect becomes more pronounced as a lower pre-biasing value $\mathrm{V}_{\mathrm{GS}}{ }^{0}$ is chosen.
After $\mathrm{V}_{\mathrm{GS}}{ }^{0}=-10 \mathrm{~V}$ it takes the drain current several seconds to reach even $1 \%$ of its saturation value. At such a heavy pre-biasing, $\mathrm{I}_{\mathrm{D}}(\mathrm{t})$ has a point of inflection indicating a second order effect. No point of infliction is observed for $\mathrm{V}_{\mathrm{GS}}{ }^{0} \geq-5 \mathrm{~V}$. The transients can be divided into two regions: A region of fast current increase $(0 \leq t \leq 1000 \mathrm{~s})$ and a region of slow current increase $(\mathrm{t}>1000 \mathrm{~s})$. These results may be explained assuming that traps are responsible for the current reduction. However, the location of the traps cannot be identified from these experiments. Charged deep levels in the region below the gate may deplete the channel of mobile carriers. Also, under large negative gate bias, the lateral field between gate and drain may lead to a charge redistribution on the surface or in the $\mathrm{AlGaN}$ barrier layer.

Assuming the existence of traps in the semiconductor or on the surface, the following simple approach can be pursued to explain the observed current reduction: If the traps can be negatively charged, this immobile charge depletes the channel and limits the drain current. As a result, after turning on the device, the channel remains highly resistive. Subsequently, the system relaxes slowly towards its equilibrium state by emission of the trapped electrons and the drain current builds up. Invoking the principle of detailed balance and neglecting hole emission from the traps, the time constant $\tau$ of the build-up process should follow the relationship [4]:

$$
\ln \left(T^{2} \tau\right)=\frac{E_{a}}{k} \cdot \frac{1}{T}-\ln \left(\frac{\sigma \bar{v} N_{c}}{T^{2}}\right)
$$

$\bar{v}$ is the thermal velocity of electrons, $\sigma$ the electron capture cross section, $\mathrm{k}$ the Boltzmann constant, $\mathrm{N}_{\mathrm{c}}$ the effective density of states in the conduction band, $\tau$ the time constant and $E_{a}$ the activation energy. In this model, the transients show an exponential behaviour:

$$
I_{D}(t)=a+b\left(1-e^{-t / \tau}\right)
$$

This simple model cannot describe the transients as a whole, but for the region of fast current increase it seems to be at least a valid approximation.

Using this approach, the transients measured at different temperatures $\left(\mathrm{RT}-280^{\circ} \mathrm{C}\right.$, Figure 4) were fitted with (Equation (2)) and the time constant $\tau$ was extracted. From the temperature dependence of $\tau$ an activation energy $E_{a}=280 \mathrm{meV}$ and a capture cross section of $4.4 \cdot 10^{-18} \mathrm{~cm}^{2}$ are extracted from the Arrheniusplot as depicted in Figure 5.

To investigate the region of slow drain current increase we studied the wavelength dependence of the steady state drain current $\mathrm{I}_{\mathrm{D}}{ }^{\infty}$. The device was biased at $\mathrm{V}_{\mathrm{GS}}=0 \mathrm{~V}$ and $\mathrm{V}_{\mathrm{DS}}=100 \mathrm{mV}$ for one day in the dark before $\mathrm{I}_{\mathrm{d}}{ }^{\infty}$ (dark) was measured. In the following the 
device was illuminated with a light intensity of $0.4 \mathrm{~W} /$ $\mathrm{m}^{2}$ with the wavelengths $\lambda=1500 \mathrm{~nm}, 1200 \mathrm{~nm}$ and the values indicated in Figure 6. The corresponding drain current was measured after 10,000 s of constant illumination. For the long wavelengths we used a halogen lamp and a monochromator for wavelength selection. A Xe-lamp was used for the short wavelengths. At $\lambda=1500$ $\mathrm{nm}$ and $1200 \mathrm{~nm}$ no significant current variation was observed. For $\lambda \leq 900 \mathrm{~nm} \mathrm{I} \mathrm{I}_{\mathrm{D}}$ increased. The energy of the onset of the current increase is well below the bandgap energy of GaN. The results indicate, that the material exhibits pronounced persistent photoconductivity (PPC).

\section{Conclusion}

It was shown that the drain current in the investigated undoped $\mathrm{AlGaN} / \mathrm{GaN}$ FETs is dependent on the gate and drain bias history. The drain current recovery is a function of temperature with an activation energy of $E_{a}=280 \mathrm{meV}$. The physical origin of the current reduction after pinch-off and its relation to the PPC is not yet understood. Further work has to be done to determine if the extracted activation energy $E_{a}$ is related to a discrete state or an emission barrier [5] [6] [7] [8]. The assumption that the PPC and the current reduction are of the same origin is under current investigation. The effect of the current reduction needs to be identified and eliminated as it may be detrimental to the large signal performance of RF power devices.

\section{REFERENCES}

[1] K. Chu, B. Green, L.F. Eastman, "AlGaN/GaN 'HEMT's for High Power Microwave Amplifiers", MRS Meeting (4/13-17, 1998), CA, USA

[2] S. T. Sheppard, K. Doverspike, W. L. Pribble, S. T. Allen, J. W. Palmour, L. T. Kehias, T. J. Jenkins, IEEE Electron Dev. Lett. 20, 161 (1999).

[3] O. Ambacher, J. Smart, J. R. Shealy, N. G. Weimann, K. Chu, M. Murphy, W. J. Schaff, L. F. Eastman, R. Dimitrov, L. Wittmer, M. Stutzmann, W. Rieger, J. Hilsenbeck, J. Appl. Phys. 85, 3222 (1999).

[4] D.K. Schroder, "Semiconductor material and device characterization", John Wiley Sons, New York (1990)

[5] A.Y. Polyakov, N.B. Smirnov, A.V. Govorkov, M. Shin, M. Skowronski, D.W. Greve, J. Appl. Phys. 84, 870-876 (1998).

[6] M. T. Hirsch, J. A. Wolk, W. Walukiewicz, E. E. Haller, Appl. Phys. Lett. 71, 1098 (1997).

[7] H. M. Chen, Y. F. Chen, M. C. Lee, M. S. Feng, J. Appl. Phys. 82, 899 (1997).

[8] C. H. Qiu, J. Y. Pankove, Appl. Phys. Lett. 70, 1983 (1997).

\section{FIGURES}

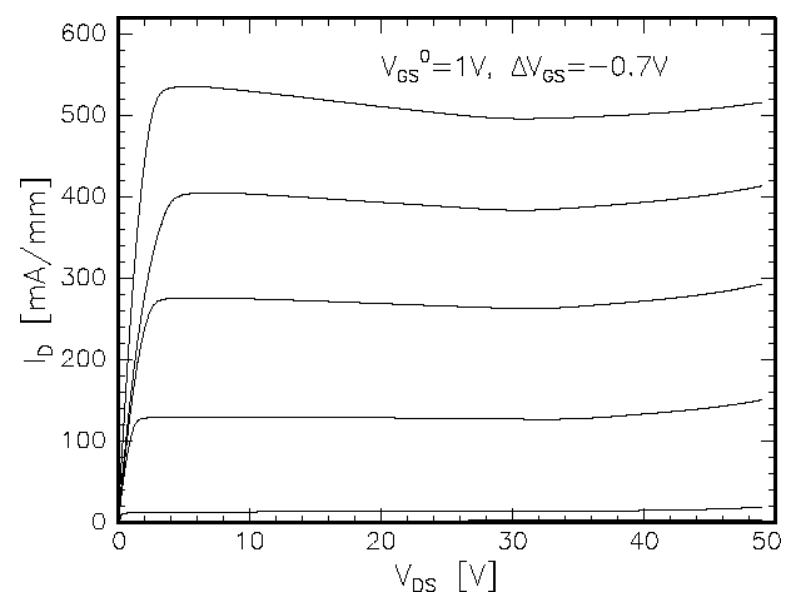

Figure 1. Output characteristic measured under UVillumination.

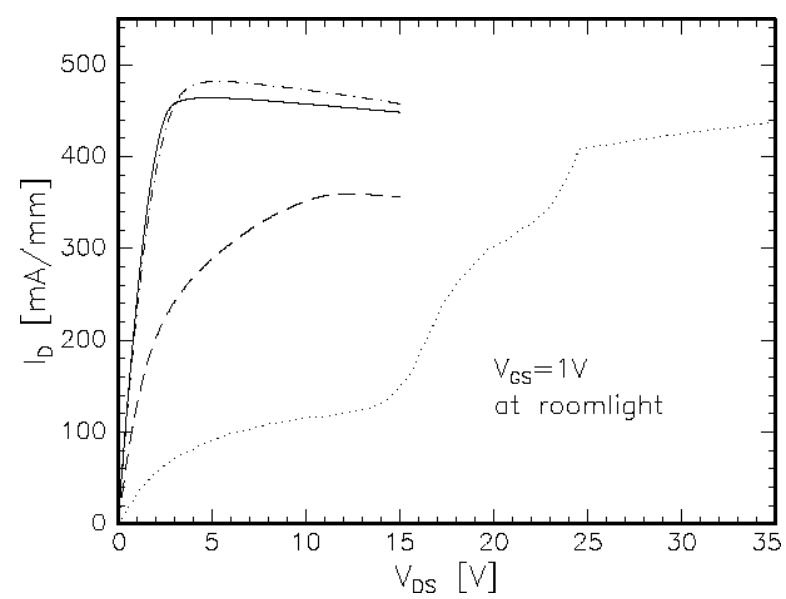

Figure 2. Topmost trace of the output characteristic for $\mathrm{V}_{\mathrm{GS}}=1 \mathrm{~V}$ : a) after several hours at roomlight and zero bias (straight line), b) 10s afterwards (dashed line), c) after brief UV-illumination (dash-dotted line). The dotted line shows the topmost trace after pinching off the device for $5 \mathrm{~min}$. 


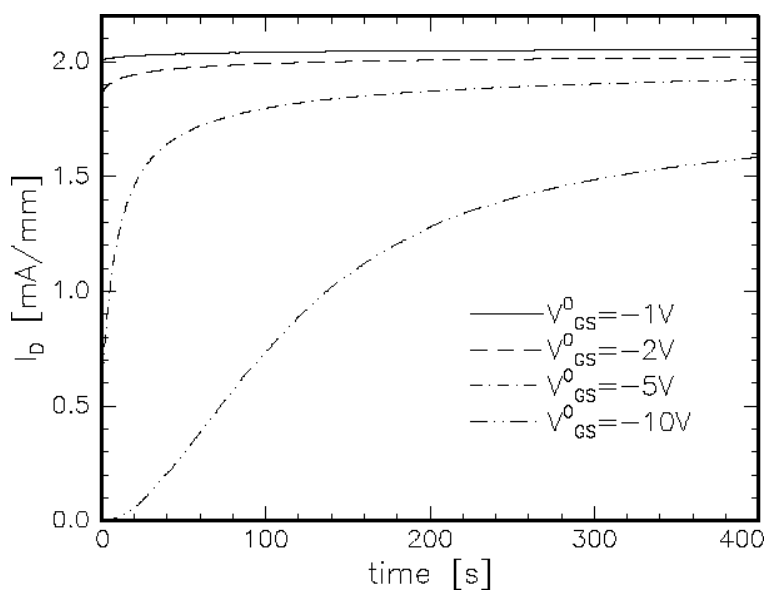

Figure 3. The first 400 s of the drain current transients for $\mathrm{V}_{\mathrm{GS}}=0 \mathrm{~V}$ and $\mathrm{V}_{\mathrm{DS}}=100 \mathrm{mV}$ measured for 5 hours in the dark. Before each transient the sample was biased at $\mathrm{V}_{\mathrm{DS}}{ }^{0}=100 \mathrm{mV}$ and the $\mathrm{V}^{0}{ }_{\mathrm{GS}}$ indicated in the figure.

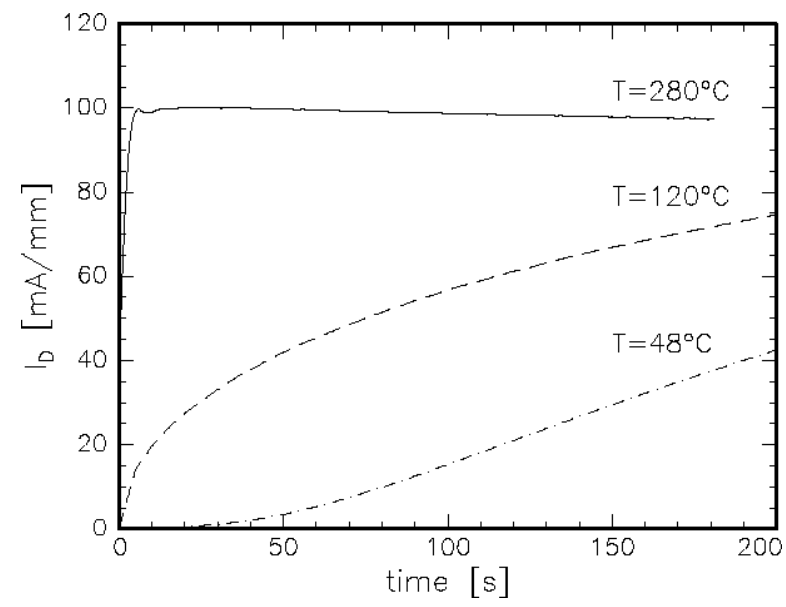

Figure 4. Drain-current transients measured at different temperatures with $\mathrm{V}_{\mathrm{GS}}=1 \mathrm{~V}$ and $\mathrm{V}_{\mathrm{DS}}=6 \mathrm{~V}$. Prior to the measurement the device was pre-biased at $\mathrm{V}_{\mathrm{GS}}{ }^{0}=-10 \mathrm{~V}$ and $\mathrm{V}_{\mathrm{DS}}{ }^{0}=6 \mathrm{~V}$ for $5 \mathrm{~min}$.

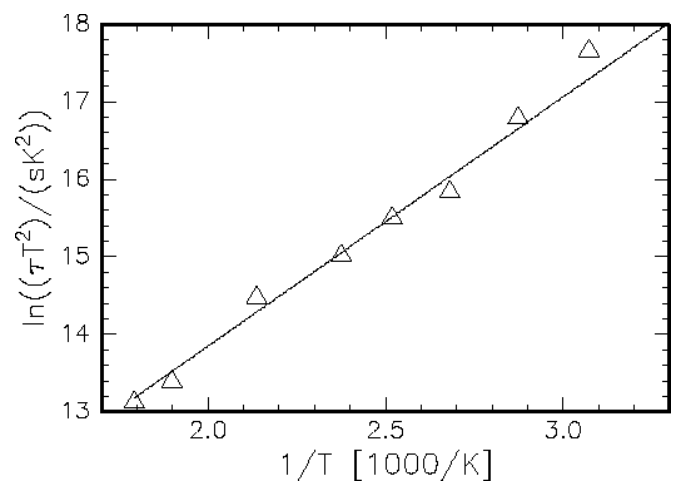

Figure 5. Arrhenius-plot of the time constants $\tau$ determined from the drain current transients measured at different temperatures.

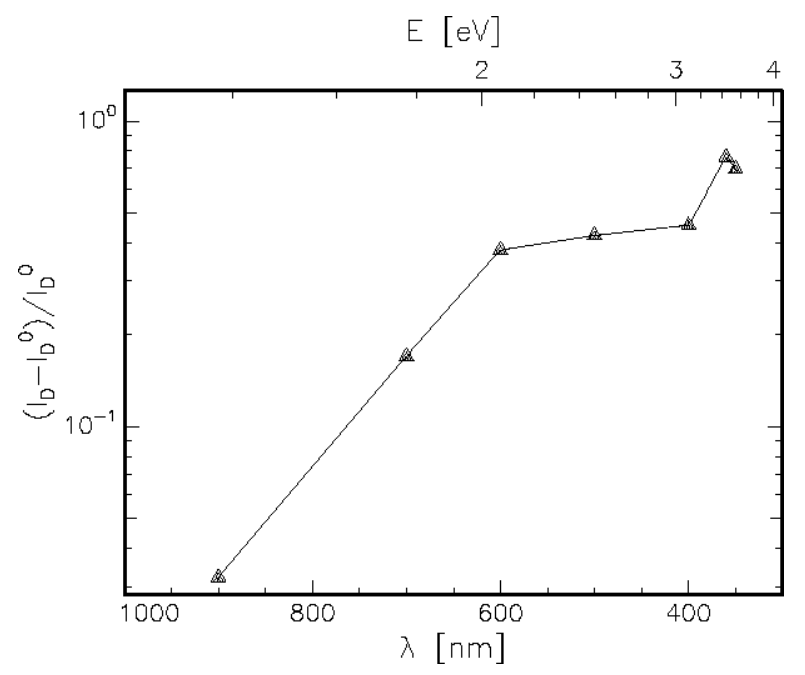

Figure 6. Steady state drain-currents for $\mathrm{V}_{\mathrm{DS}}=100 \mathrm{mV}$ and $\mathrm{V}_{\mathrm{GS}}=0 \mathrm{~V}$. The light intensity is $0.4 \mathrm{~W} / \mathrm{m}^{2}$. 